\title{
A High-Speed Electron-Counting Direct Detection Camera for TEM
}

\author{
P.E. Mooney*, D. Contarato**, P. Denes**, A.J. Gubbens*, B. Lee*, M. Lent*, D.A. Agard*** \\ *Gatan, Inc., 5794 W. Las Positas Blvd, Pleasanton, CA 94588 \\ **Lawrence Berkeley National Laboratory, 1 Cyclotron Rd, Berkeley, CA 94720 \\ ***Biochemistry and Biophysics Dept., UCSF, 600 16th St, San Francisco, CA 94158
}

It has long been known that electron scatter is the main source of signal degradation in imaging detectors for electron microscopy. Silicon and more specifically, the silicon active pixel sensor (APS), shows promise as a way to address this fundamental signal quality bottleneck. As can be seen in figure 1, scatter from the lower atomic number silicon detection medium (1b) creates a much narrower cone of tracks than the higher atomic number scintillator/fiber optic assembly (1a). Because the signal is taken out of the detection medium by direct electronic readout and not by optical transfer, the detector can be thinned, greatly reducing backscatter. In addition, the energy collection resolution is increased and the signal to noise ratio dramatically raised. The last benefit in turn allows discrimination of incoming electron events and centroiding of the electron's entry position which provides additional noise reduction and resolution improvement. Gatan, in collaboration with the Lawrence Berkeley National Laboratory and UCSF and with support from the Howard Hughes Medical Institute and the National Science Foundation is developing a direct detection camera based on a thinned APS.

Direct detection places a requirement for radiation hardness on the detector which has hitherto been protected by the fiber optic image transfer plate or by physical separation through lens coupling. APSs are intrinsically more radiation hard than CCDs but additional measures have been taken in recent years to greatly extend the radiation hardness for purposes of high energy radiation detection [1] and more recently electron microscopy [2]. A single electron counting and centroiding capability place an additional requirement of speed on the camera system. A high frame rate is needed to allow sufficient event separation. The high speed in turn generates a very high data rate. In order for the camera to be useful with conventional computer hardware, builtin processing is needed to calculate the centroids and accumulate event-based images for transfer to a host computer at conventional data rates.

The camera under development will have approximately $4 \mathrm{k}$ x $4 \mathrm{k} 5 \mu \mathrm{m}$ pixels manufactured in $0.18 \mu \mathrm{m}$ CMOS technology for maximum radiation hardness, will be thinned to minimize electron backscatter and will be read out at 400 frames per second for processing. A processing unit will allow accumulation and transfer to the host at rates up to $40 \mathrm{fps}$. Optionally, the processing unit can be left out, still allowing simple linear accumulation. The $5 \mu \mathrm{m}$ pixel size was chosen based on feasibility experiments performed on a candidate area of a test sensor [3] with 152 x 192 pixels. Figure 2 shows resolution results from the test sensor, both linear (red curve) and using centroid processing (blue) alongside DQE from the $14 \mu \mathrm{m}$ pixel US1000, confirming expectations for a dramatic increase in DQE both from the thinned APS architecture alone and from processing. The DQE beyond the Nyquist frequency is comparable with a fiberoptically coupled scintillator just above half the Nyquist frequency. The test sensor also confirmed radiation hardness of at least $5 \mathrm{Ge}$ - per pixel with extensions well beyond that expected with cooling to $-20 \mathrm{C}$ and the $8 \mathrm{x}$ faster integration time of the final camera. 
The full camera is near completion. Figure 3 shows the first light image of an optically projected air force resolution target from a 512 x 1024 subarea of the full-size detector. The $4 \mathrm{k} \times 4 \mathrm{k}$ direct detection camera with centroided, upsampled accumulation to an effective $8 \mathrm{k}$ x $8 \mathrm{k}$ resolution, will provide a major performance leap for image acquisition in low-dose applications such as structural biology and cryo-tomography. The extremely high frame rate possible in subarea and binned modes in conjunction with the high sensitivity will constitute a major advance in in situ video acquisition as well.

\section{References:}

[1] R. Turchetta et al., Nucl. Instr. and Meth. A 458 (2001) 677.

[2] P. Denes et al., Nucl. Instr. and Meth. A 579 (2007) 891.

[3] D. Contarato, 2011, in press. DOI 10.1016/j.nima.2011.01.087

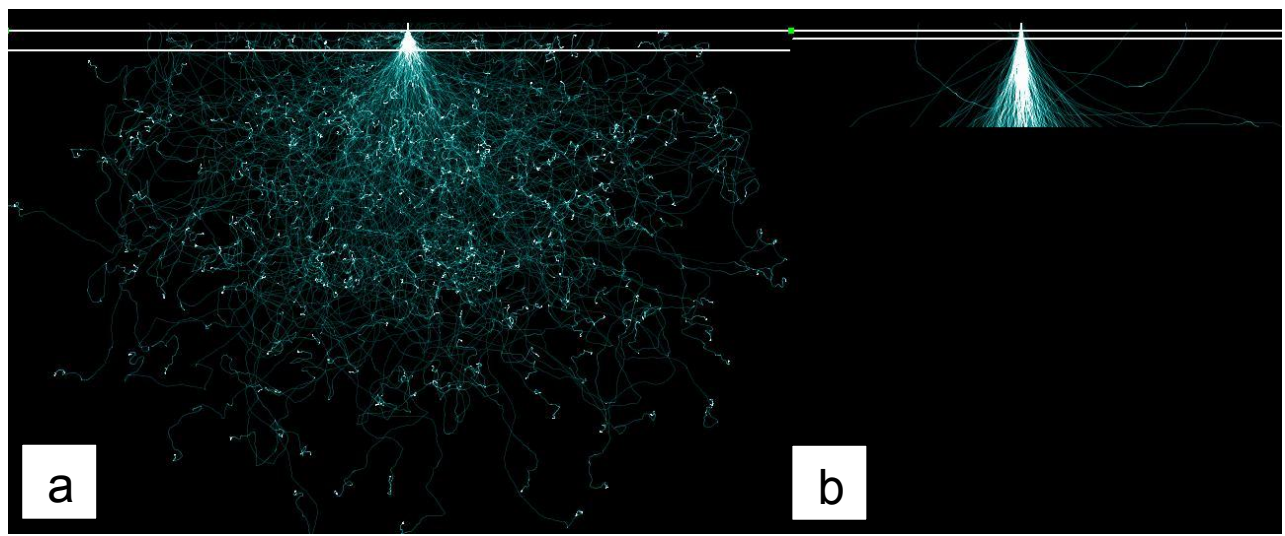

FIG. 1. Monte Carlo simulations of $300 \mathrm{kV}$ electrons impinging on a scintillator-fiber optic assembly (a) and on a thinned silicon APS (b). Lines are drawn above and below the energy sensitive volumes in both cases: scintillator in (a) and epitaxial layer in (b). The simulation software was provided by Raynald Gauvin, McGill University.

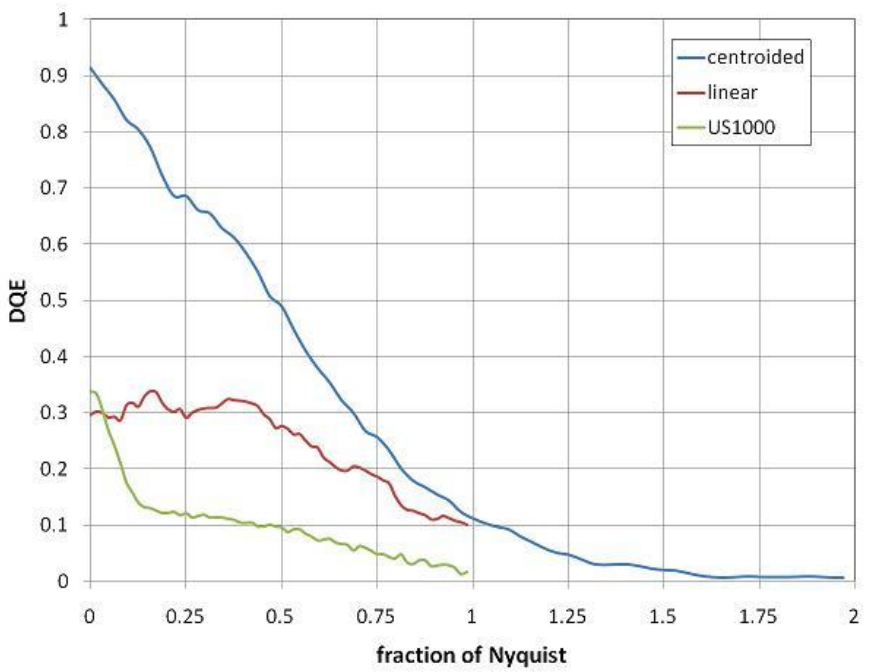

FIG. 2. DQE of the $152 \times 192$ test sensor. The middle curve is the DQE with linear accumulation. The top curve is the DQE with centroided single electron event processsing. The bottom curve is the US1000 for comparison.

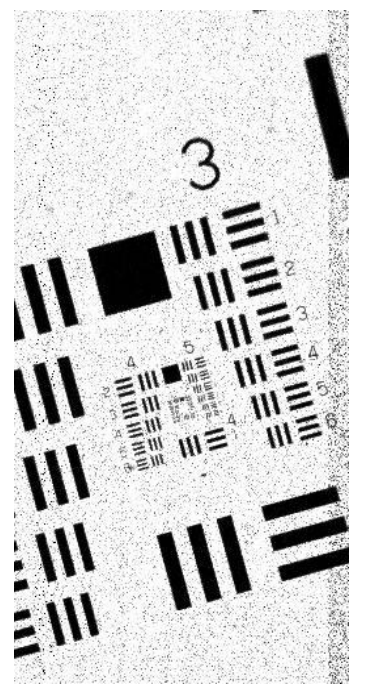

FIG 3. The first light image from the full-sized sensor showing a $512 \times 1024$ pixel subarea. 